\title{
System-level design of a 64-channel low power neural spike recording sensor
}

\author{
Manuel Delgado-Restituto, Senior Member, IEEE, Alberto Rodríguez-Pérez Member, IEEE, Angela Darie, \\ Cristina Soto-Sánchez, Eduardo Fernández-Jover and Ángel Rodríguez-Vázquez, Fellow, IEEE
}

\begin{abstract}
This paper reports an integrated 64-channel neural spike recording sensor, together with all the circuitry to process and configure the channels, process the neural data, transmit via a wireless link the information and receive the required instructions. Neural signals are acquired, filtered, digitized and compressed in the channels. Additionally, each channel implements an auto-calibration algorithm which individually configures the transfer characteristics of the recording site. The system has two transmission modes; in one case the information captured by the channels is sent as uncompressed raw data; in the other, feature vectors extracted from the detected neural spikes are released. Data streams coming from the channels are serialized by the embedded digital processor. Experimental results, including in vivo measurements, show that the power consumption of the complete system is lower than $330 \mu \mathrm{W}$.
\end{abstract}

Index Terms-Neural prosthesis, Spike detection, BrainMachine Interfaces (BMI), Neural recording, Feature extraction

\section{INTRODUCTION}

In recent years, advances in technology have made it possible to monitor bioelectric activity using neural prosthesis implanted in the brain [1]-[4]. These devices obtain much higher spatio-temporal resolution than using electroencephalography (EEG) techniques, thus allowing specialists to prescribe more specific treatments and even to develop new therapeutic procedures. The potential of neural prostheses is particularly noticeable when they are tailored to the detection and analysis of Action Potentials (APs, also called spikes). For instance, changes on the neural spiking activity during the different phases of epilepsy could eventually serve as a biomarker for seizure prediction [5], [6]. Implanted closed loop neuromodulation systems based on spike identification could be a promising solution for counteracting the adverse effects of certain illnesses, such as the motor dysfunction caused by Parkinson's disease [7]. By looking at the spiking activity recorded in hippocampal ensembles, memory prosthesis devices can be designed to enhance short-term memory in real-time for patients with memory disorders that result from head injury or chronic deterioration such as Alzheimer's

This work has been supported by the Spanish Ministry of Economy \& Competitiviness under grant TEC2016-80923-P, the ONR under grant N000141110312 and the FEDER Program.

Manuel Delgado-Restituto, Alberto Rodríguez-Pérez, Angela Darie, and Ángel Rodríguez-Vázquez are with the Institute of Microelectronics of Sevilla (IMSE), University of Sevilla - CSIC, Sevilla, SPAIN (e-mail: \{mandel, alberto, angela, angel\} @imse-cnm.csic.es). Cristina Soto-Sánchez, Eduardo Fernández-Jover are with the Bioengineering Institute, University Miguel Hernandez and CIBER-BBN, Elche, SPAIN (e-mail: \{csoto, e.fernandez\}@goumh.umh.es). disease. [8]. Additionally, the efficient monitoring of spike trains is key for the development of Brain-Machine Interfaces (BMI) in which appropriately instrumented objects can be controlled exclusively by a person's thoughts, thus improving the quality of life of patients with severe immobility problems as, for instance, after a spinal cord injury [9]-[11].

As long as these neural monitoring systems are implanted, they have to achieve and maintain stable long-term recordings so that the need for re-surgery is essentially eliminated. This poses important challenges on the hardware implementation:

- On the one hand channel pitches and system form factors must be small. On the other, channels and systems must be versatile and adaptable. Versatility is needed to cope with different monitoring setups as determined by neurologists. Adaptability is needed to deal not only with the intrinsic statistical deviations of the fabrication process but also with the non-stationary nature of the electrode-tissue interface.

- Power consumption must be small to prevent from harmful effects due to excessive heating and to minimize energy requirements. This latter aspect is particularly relevant for remotely powered, battery-less implants.

- Data compression techniques are needed to reduce the power budget of information processing and communications. In the case of neural spike recorders, each sensor should idle and does not process any information unless the event of a neural spike is detected. The ultimate limit of data compression is not to undermine the efficiency of the therapeutic procedure or the brain-machine interface in which the recording system is embedded. For instance, in BMIs, not only the presence of a spike but also information about its morphology must also be provided so that action potentials can be properly sorted. Further, the temporal information of the events must be preserved along the entire recording period.

Several remarkable integrated low power neural monitoring systems have been proposed during the last years [12]-[27]. Many of them feature wireless telemetry and remote powering functionalities. In some cases, bandwidth reduction techniques based on the detection and compression of neural spikes have been proposed as well [15], [21], [23]-[26]. However, existing proposals have only partially addressed the adaptability of the recording system to the plastic behavior of the neural tissue or the alterations in the galvanic contact at the electrodes.

In this work, an integrated 64-channel neural spike recording System-on-Chip (SoC) is presented. The main objec- 


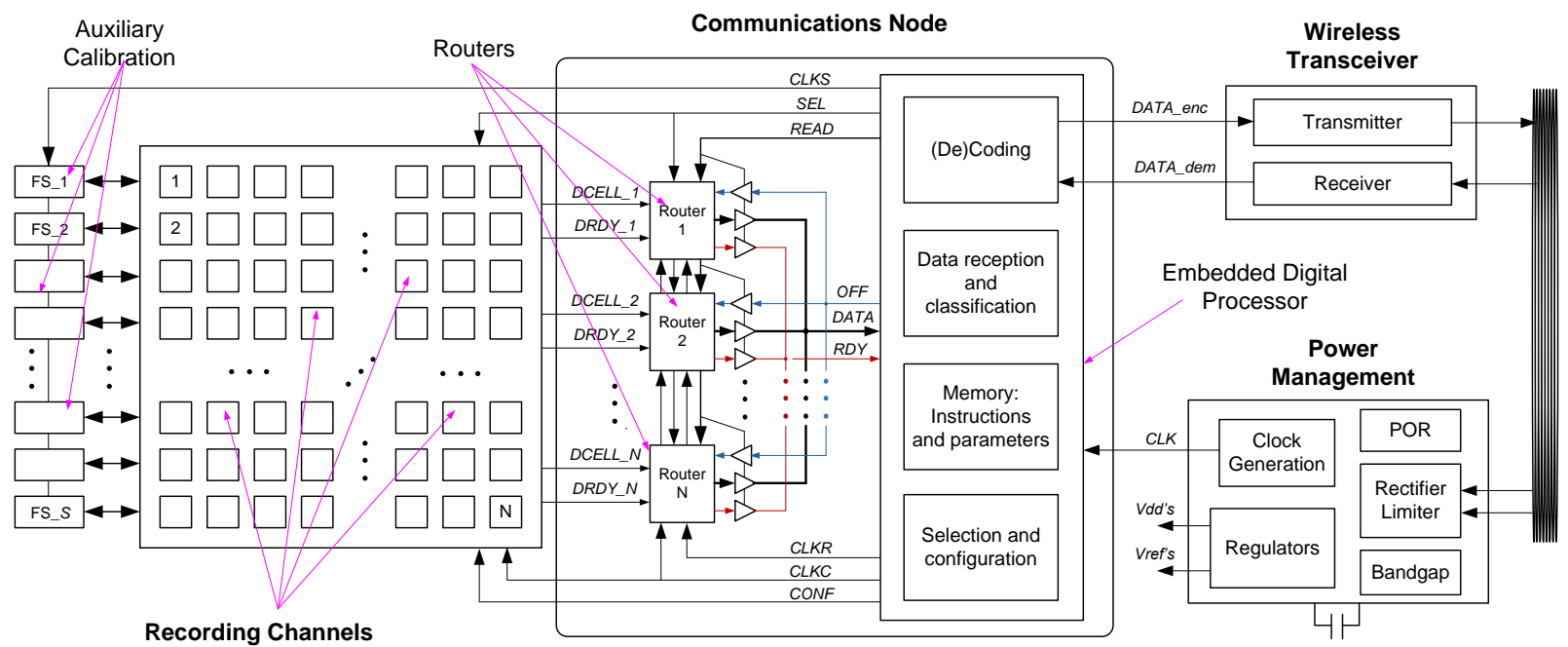

Figure 1. Architecture of the neural recording SoC.

tive has been to provide techniques for long-life operation without penalizing its power consumption or increasing its area occupation. Some of the distinctive features of this SoC are: (i) it implements a bi-directional communication link not only to retrieve information about the brain activity but also to interact with the implant itself without the need of surgery intervention; (ii) two different transmission modes, uncompressed raw data or feature vectors of detected spikes, can externally selected on demand; (iii) channels can be individually disabled if the associated sensor does not transmit any relevant information or the tissue-electrode interface is completely degraded, thus reducing power consumption; (iv) the analog front-ends of the recording channels include programming and updating solutions for the automatic (or at least, externally-controlled) adjustment of their individual pass-band characteristics; (v) the identification of neural spikes, which ultimately relies on the adjustment of an empirical threshold [28], takes into account the spike-to-noise ratio of the captured signal and employs an adaptive algorithm for improving the probability of detection; (iv) the feature extraction of neural spikes operates in real-time and obtains similar classification performance as other more sophisticated techniques with no need for dimensionality reduction pre-processing [28]. All these features make the proposed neural spike recording SoC an observation tool which allows neurologists to examine the state of the implant and eventually reconfigure the array of sensors to better serve the purposes of the application at hand.

This paper extends the results in [29] and describes the acquisition and communications sections of the SoC, the partitioning of the signal processing tasks, and the structure of the digitally-assisted neural recording channels. Additionally, it describes and experimentally demonstrates the programming and self-adaptation techniques embedded in the SoC, assesses the accuracy of the implemented data reduction algorithms and includes in vivo measurements under raw data and feature extraction modes.

The architecture of the recording SoC as well as a description of the different operation modes are presented in Sec. II.
Afterward, Sec. III describes the embedded micro-controller in the communications node of the system. After disclosing the communication protocol in Sec. IV, the paper presents in Sec. $\mathrm{V}$ the architecture of the recording channels and the digital control module therein. Then, Sec. VI gives a brief overview of the analog front-end of the channels. Section VII illustrates the operation of the SoC, provides experimental measurements and in vivo validation results and compares the proposed recording system to others in the current state-ofthe-art. Finally, Sec. VIII concludes the paper.

\section{NEURAL RECORDING SOC ARCHITECTURE}

Fig. 1 shows the proposed SoC architecture. It includes a communications node, which acts as gateway between a set of $N=64$ recording sites and a wireless transceiver. Both remote powering and wireless data transfer are implemented through a single inductive link to an external hub placed on the head. The approach is largely inspired in RFID techniques [30] and uses the worldwide available ISM (industrial, scientific and medical) band centered at $40.62 \mathrm{MHz}$ for energy/data transmission. On-Off Keying (OOK) modulation is used to transmit data and power from the external hub to the SoC and load shift keying (LSK) modulation is used to transmit data in the reverse direction. The SoC also embeds a power management block, and a set of $S=8$ frequency synthesizers (FS) for adjusting the data transfer characteristics of the recording channels. The power consumption of the wireless transceiver and the power management blocks amounts approximately $47 \mu \mathrm{W}$. All the blocks in Fig. 1 are fully integrated on-chip and are individually accessible through dedicated pins for testing purposes. In this paper, focus is put on the description of the communications and recording blocks - details on the remote powering and wireless data transfer will be reported elsewhere.

The recording channels, distributed in a $8 \times 8$ array, amplify and filter the neural signals captured from the electrodes and include circuitry to automatically calibrate the output voltage level and bandwidth of the recorded signals. Analog-to-digital 
conversion and information compression of detected neural action potentials are other functions realized in-channel.

The communications node includes a dedicated MicroController Unit (MCU) for: (i) interpreting and applying the commands received from the wireless transceiver; (ii) digitally processing the signals provided by the recording channels; and (iii) encoding the information collected from these sensors for transmission through the wireless link. The communications node includes $N$ signal routers, one per channel, which essentially work as data rate up-converters for raising the transfer speed of the channels to the master clock rate of the MCU. In this work, a custom MCU, instead of an external commercial unit or an embedded IP solution, has been chosen in order to save power and area consumptions, as well as to reduce the form factor of the whole SoC.

The commands interpretable by the MCU fall into one of four categories, each category associated with an operating mode, MODE. They are:

1) Configuration mode $(M O D E=' 00$ '), in which the operating parameters of the recording channels are assigned.

2) Calibration mode $(M O D E=$ ' 01 '), in which the passband and gain of the transfer characteristics of the channels are automatically adjusted. This facility provides a convenient solution for counteracting inter- and intradie process variations, as well as, for adapting against changes in the working conditions of the implant like, for instance, tissue-electrode interface degradation or electrode displacement.

3) Signal Monitoring mode $\left(M O D E={ }^{\prime} 10 '\right)$, in which the signals captured by the selected channels are transferred in raw format to the MCU. In this mode, the output stream generated by the MCU contains recordings from a single row/column of channels; all these recordings acquired at the same instant. The selected row/channel may remain the same or be part of a subset of rows/columns which are cyclically visited. As the master clock rate of the communications node is fixed, this poses a trade-off between the number of channels being read out and the effective throughput rate per channel.

4) Data Compression mode $(M O D E=' 11$ '), in which neural action potentials are detected and characterized in real-time by a reduced set of $F$ representative parameters, $F E \_D A T A$, in order to reduce the bandwidth of the transmitted signal. Data output from a recording channel remains idle if no spike is detected.

Although different channels in a selected subset may have different parameters, they all have to run in the same operation mode. Mixed configurations with some channels operating in e.g., Signal Monitoring mode, and some others in, e.g. Data Compression mode, are not allowed.

\section{DEDICATED MCU}

Fig. 2 shows the structure of the dedicated MCU. It operates from a $4.0 \mathrm{MHz}$ master clock $C L K$ which is internally scaled in frequency for driving other elements in the SoC, namely, it generates: (i) signal $C L K R$ for the routers and the shaded elements in Fig. 2 (same rate as $C L K$ ); (ii) $C L K C$ for the recording channels and the routers ( $1 / 5 C L K R$ rate); (iii) $C L K M$ for the non-shaded digital blocks in the MCU ( $1 / 3 C L K C$ rate); and (iv) $C L K S$ for the calibration units $(1 / 7 C L K C$ rate).

During command reception, the data demodulated by the receiver is aligned with clock $C L K R$ and decoded from Pulse Interval Encoding (PIE) format to binary code. This information is stored in a register and a CRC (Cyclic Redundancy Check) block verifies the data integrity. A command ID block analyzes the content of the register and identifies the operation mode MODE specified for the SoC. Then, a finitestate machine (FSM_Rx, one per operation mode) is used for identifying the command option, its range of application and the configuration parameters, if available, comprised in the received instruction. This information is ultimately used by a Selection and Programming block to generate the output vectors $C O N F$ and $S E L$ which define the states of the signal routers and the recording channels. The $N$-bit $S E L$ vector, one bit per recording channel, is used to selectively activate the channels of the array. The CONF vector is used to serially load operating parameters to those channels addressed by $S E L$.

For data transmission, a finite-state machine (FSM_Tx, one per operation mode) is used for reading, processing and sending the data stored in the selected routers to the encoding section, formed by a parallel-series converter and a Manchester code converter. The information DATA gathered by the routers is retrieved by the MCU by means of tri-state buffers controlled by a pointer $R E A D$, which cyclically scans the selected routers. If pointer $R E A D$ addresses a router with no information to transmit (signal $R D Y$ in low logic state), it jumps to the next active router. Otherwise, if signal $R D Y$ is in high logic state, DATA is transferred to the MCU and, afterward, RDY turns to low state by the action of pulse $O F F$. This data retrieval approach supports the event-based transmission of neural spikes in the Data Compression mode. Recording channels in the subset $S E L$ remain in idle state and do not transmit any information unless a neural spike has been detected and characterized.

The MCU also comprises a data stack in which global parameters (affecting the whole array) are recorded, overwriting any previous data, and a ROM in which default values are stored. When the SoC is switched on, the data stack loads the values stored in such non-volatile memory, which are then transferred to the recording channels. It is also possible to reload the content of the ROM at any moment by using the appropriate command.

Power saving has been one of the main challenges accounted for in the implementation of the MCU. All those blocks not addressed by $S E L$ or enabled by $M O D E$ enter in a power-down state and essentially consume no energy. Furthermore, the use of multiple clocks per block and state also helps to reduce the overall dynamic power consumption of the MCU. Another important measure taken to reduce the power consumption has been the adopted signal processing partitioning strategy. Heavy computational tasks in the Calibration and Data Compression modes are run locally at the channel-level and, hence, distributed across the sensor array. One the one hand, this allows to employ lower clock frequencies than if the same tasks were 


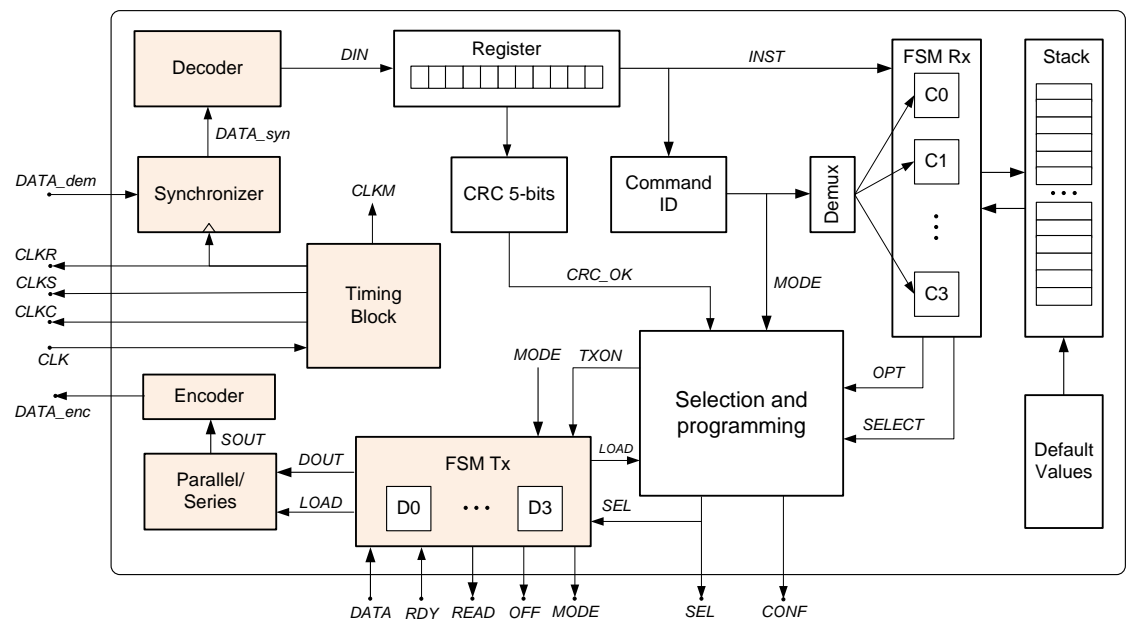

Figure 2. Architecture of the dedicated MCU.

run at the MCU and, on the other, it reduces the transfer rate between the recording channels and the MCU.

The area occupation of the MCU, including all the circuit elements in Fig. 2, is about $1.6 \mathrm{~mm}^{2}$ and its average power consumption is $40 \mu \mathrm{W}$.

\section{COMMUNICATION PROTOCOL}

Fig. 3 shows the command frames recognizable by the SoC, arranged by operation mode. Numbers between parentheses represent the bit lengths of the different fields. In most cases, frames comprise six fields: preamble; operation mode, $M O D E$; range of application of the instruction; command option, $Q E R S$ (depending on the operation mode, $Q E R S$ is identified as $C F_{-} Q, C A L_{-} Q, T R_{-} Q$ o $\left.C M P_{-} Q\right)$; set of configuration parameters PARAMS, and CRC code. Different commands may correspond to a given operation mode. They are distinguished by means of QERS which, in some cases, may address parameter groupings (as in the Configuration mode) or define operating options (as in the Signal Monitoring and Data Compression modes). The vector CONF supplied to the recording channels is composed by the fields $M O D E, Q E R S$ and PARAMS, if available.

Typically, the range of application, SELECT, can address one single recording channel (identified by code $I D \_c e l l$ ), a whole row or column in the array (identified by codes $I D \_r o w$ and $I D \_c o l$ respectively), or the totality of sensors. However, depending on the operation mode, not all ranges of application are permitted. For example, as calibration units are shared per rows (see Fig. 1), the Calibration mode may only be executed by columns or by individual sensors.

Bits in vector SEL are set to high logic state if corresponding channels are addressed by vector SELECT. In the Signal Monitoring mode, the selection range can be potentially extended by the action of $T R \_Q$.

Parameters, PARAMS, are only specified in the Configuration and Calibration modes. They are classified into $C A L \_P A R$, $T R \_P A R$ and $C M P \_P A R$ depending on whether they are related to the Calibration, Signal Monitoring or Data compression modes, respectively (more details in Sec. V).

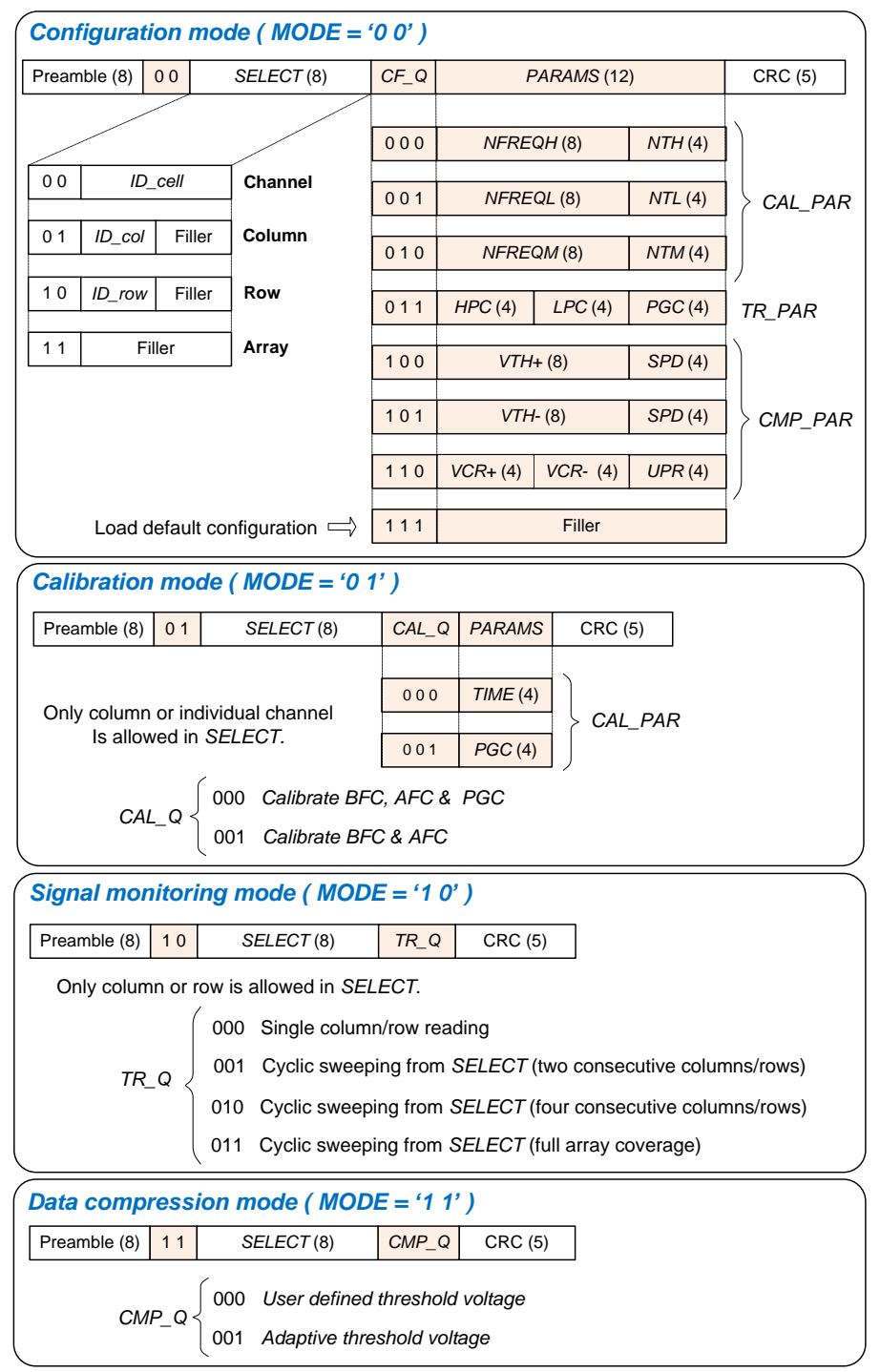

Figure 3. Recognizable input commands of the SoC. Shaded fields compose the $C O N F$ vector supplied to the recording channels. 


\begin{tabular}{|c|c|c|c|c|}
\hline \multicolumn{2}{|c|}{ Preamble (8) } & & Payload (64) & CRC (8) \\
\hline \multicolumn{3}{|c|}{ Configuration mode } & & \\
\hline \begin{tabular}{|l|l|}
00 \\
\end{tabular} & ID_cell & CONFIRM (8) & & \\
\hline \multicolumn{4}{|c|}{ Calibration mode } & \\
\hline 01 & ID_cell & \begin{tabular}{|l|l|}
$H P C(4)$ & $L P C(4)$ \\
\end{tabular} & \begin{tabular}{|l|l|}
$P G C$ (4) & filler (4) \\
\end{tabular} & \\
\hline \multicolumn{5}{|c|}{ Signal monitoring mode } \\
\hline 10 & ID_row/col & $R C \_D A T A_{1}(8)$ & $R C \_D A T A_{2}(8)$ & $R C_{-} D A T A_{K}(8)$ \\
\hline \multicolumn{5}{|c|}{ Data compression mode } \\
\hline 11 & ID_cell & $F E \_D A T A_{1}(8)$ & $F E_{-} D A T A_{2}(8)$ & $F E_{-} D A T A_{F}(8)$ \\
\hline
\end{tabular}

Figure 4. Output data frames of the SoC. Shaded fields are provided from a single recording channel.

Fig. 4 shows the structure of the output data frames of the SoC for the different operation modes. In all cases, the frame begins with a preamble, followed by a data payload of fixed length (64 bits) and a CRC code. The data payload carries the information provided by the channels. Fillers are appended if the channel's response is shorter than 64 bits. This happens, for instance, in the Configuration mode, in which the recording channel replies with a short confirmation code, CONFIRM, after loading vector CONF. Similarly, in the Calibration mode, the recording channel responds with the final value of the adjusted parameters $T R \_P A R$ once the calibration process has been completed.

The Signal Monitoring mode is the only operation category in which data from different recording channels are combined in the output frame. In this mode, the data payload contains $K=8$ sample values $R C_{-} D A T A x, x=1, \ldots, K$, coming respectively from the $K$ recording channels composing a row or column in the array. These values are arranged in the frame in the same order than the channels in the array. Contrary to the other operation modes in which the identifier address a cell (ID_cell), in the Signal Monitoring mode the identifier refers to a row (ID_row) or column (ID_col) as corresponds.

In the Data Compression mode, the output frame gathers the representative values $F E \_D A T A y, y=1, \ldots, F$, obtained from the characterization of the neural action potential detected by the recording channel identified by code $I D \_c e l l$. As will be shown in Sec. V.C, depending on the command option $C M P_{-} Q$, only $F=5$ or $F=6$ parameters are needed to fully characterize an action potential.

\section{NEURAL RECORDING CHANNEL STRUCTURE}

Fig. 5 shows the block diagram of the recording channels implemented in the SoC. Each channel embeds: (i) a lownoise amplifier (LNA); (ii) a programmable variable gain amplifier (PGA) for adjusting the voltage levels provided by the LNA; (iii) an analog-to-digital converter (ADC) for digitizing the signal provided by the PGA; and (iv) a control module which identifies the operation mode of the recording channel, configures the parameters of the LNA and PGA, processes the data $R C_{-} D A T A$ digitized by the ADC, and handles the operation of a frequency synthesizer (shared by columns in the array) during calibration. In the Calibration

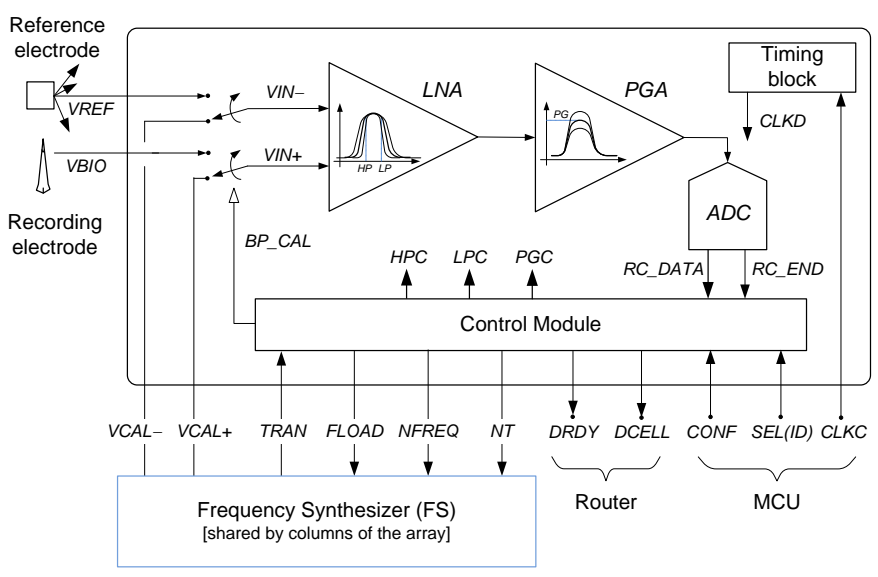

Figure 5. Recording channel architecture. The frequency synthesizer is not part of the recording channel but it is placed in the periphery of the channel array.

and Signal Monitoring modes, the recording channel operates at a rate $C L K D$, obtained by a local timing block which divides the incoming clock signal $C L K C$ by three. In the Data Compression mode, two phases can be distinguished. When the channel is scanning the captured neural signal for detecting spikes, it also uses the rate CLKD, however, when detected neural APs are being processed, the operation rate, including the ADC sampling speed, is directly given by $C L K C$.

The LNA exhibits a bandpass transfer characteristic whose High-Pass (HP) and Low-Pass (LP) cut-off frequencies can be digitally programmed by means of control words $H P C<1: N H P>$ and $L P C<1: N L P>$, respectively. Likewise, the gain of the PGA can be controlled by using the digital word $P G C<1: N P G>$. Circuit details on the Analog Front-End (AFE) are presented in Sec. VI. Hereafter, focus is on the design of the control module embedded per channel, whose block diagram is shown in Fig. 6.

The control module comprises: (i) an command reading block; a parameter storage facility in which the channel configuration is archived; (ii) three blocks which execute instructions related to the Calibration mode, Signal Monitoring mode and Data Compression mode, respectively; and (iii) a data transmission module which builds the output DCELL of the recording channel. The command reading block indexes the content of the configuration vector $C O N F$ by identifying the operating mode $M O D E$, the command options $Q E R S$, and the associated parameters PARAMS, if any.

Note that the Configuration mode needs no specific instruction block, as parameters PARAMS are directly transferred to the storage memory. The other instruction blocks are separately described below.

\section{A. Calibration block}

The purpose of the calibration block is to automatically program the set of parameters $T R \_P A R$, comprising vectors $H P C, L P C$ and $P G C$, so that the transfer characteristics of the AFE meets the target passband. If no calibration is run, the recording channel uses the parameters $T R \_P A R$ stored in the local memory. As noted in Fig. 3, vector $P G C$ can be either 


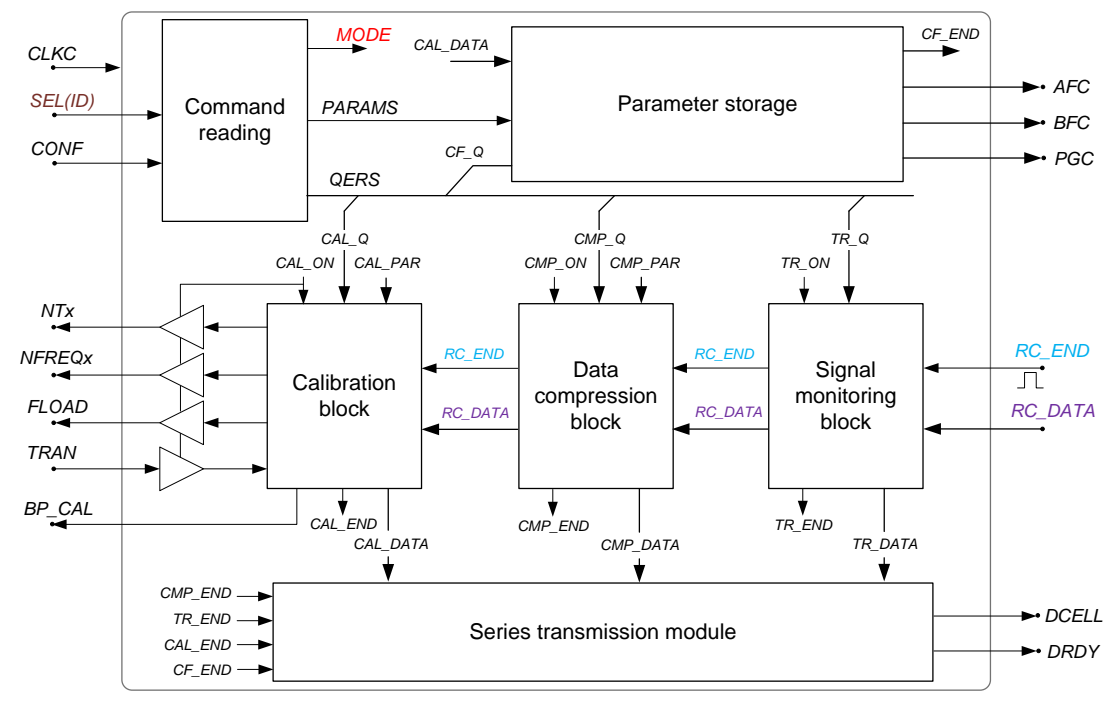

Figure 6. Block diagram of the control module included in the recording channels.

automatically trimmed or fixed to an externally defined value, depending on the command option $C A L \_Q$.

The tuning approach for the adjustment of the HP and LP channel corners is based on a mixed-signal control loop which uses, as feedback signal, the output of the ADC (see Fig. 5) and, as controlled signals, the vectors $H P C$ and $L P C$, respectively. The approach relies on peak amplitude inspection of the ADC output and requires an auxiliary frequency synthesizer for the generation of three pilot tones. During the automatic tuning, the LNA inputs are directly driven by the frequency synthesizer and, hence, disconnected from the electrodes. The frequency synthesizer consists of a Digitally Controlled Oscillator (DCO) operated at a clock rate CLKS, followed by a MOS-based R-2R current steering DAC, and a first order low-pass smoothing filter [31].

Data transfer between the calibration block and the frequency synthesizer (FS) uses tri-state buffers. The frequency of the pilot tones is defined by digital words NFREQx ( $x$ stands for $H, L$ or $M$ ), which are loaded at instances of a pulse FLOAD. For each NFREQx, a number of tone cycles $N T x$ is used as an estimation of the transient period required by the calibration loop to settle. Once the transient period is over, the calibration block defines a measurement period which occupies $N M=N T x$ periods of the synthesized tone.

The foreground automatic tuning of the HP and LP poles encompasses three steps:

1) Control words $H P C$ and $L P C$ are initially set to the widest bandpath possible and the pilot tone, defined by NFREQM, is an arbitrary frequency within the target passband characteristic. After a PGA calibration process to maximize the ADC input swing, the calibration block stores the digital peak amplitude $V_{a}$ detected at the ADC output in the measurement period.

2) Control word $H P C$ is set to the highest frequency possible and the pilot tone, defined by $N F R E Q H$, is made to coincide to the intended HP pole position. The calibration block launches a searching process which aims to identify that HPC configuration which minimizes
$\left|V_{a, H P}-\alpha \cdot V_{a}\right|$, where $V_{a, H P}$ is the peak amplitude of the ADC output during HP calibration and $\alpha$ is a scaling factor ( $\alpha=3 / 4$ has been implemented).

3) Control word $L P C$ is set to the lowest frequency possible and the pilot tone, defined by $N F R E Q L$, is made to coincide to the intended LP pole position. A similar searching procedure as in step 2 obtains the $L P C$ code that more closely approximates the intended LP pole.

During the adjustment of the PGA, the LNA inputs are connected to the electrodes. The calibration starts by setting the PGA to its maximum gain value and calculating the maximum and minimum amplitudes at the output of the ADC during a time interval specified in parameter TIME. If any of these amplitudes exceeds the full-scale of the ADC, the digital control $P G C$ is reduced by one. This process is repeated until no exceeding values are detected. At this point, the last control word $P G C$ is stored.

\section{B. Signal monitoring block}

The signal monitoring block essentially implements a selective pathway between the ADC of the recording channel and the data transmission block of the control module. Depending on the command option $T R \_Q$, not all vectors $R C_{-} D A T A$ are transferred to the associated router. Indeed, this only happens in the conmfiguration TR_Q = '000', in which only a single row/column is read. When $T R \_Q=$ ' 001 ' and two consecutive rows/columns are addressed, the block only passes one data conversion out of two. Similarly, when $T R \_Q=$ '010' one out of four vectors is transferred and, when $T R \_Q=$ ' 011 ' and the whole array is cyclically swept, only one out of eight $R C \_D A T A$ reaches the data transmission module. In practice, this implies a trade-off between the number of channels being monitored and the overall throughput rate per channel which scales down every time the number of channels is doubled. In all the configurations, the sampling rate of the channels is about $30 \mathrm{kS} / \mathrm{s}$. 


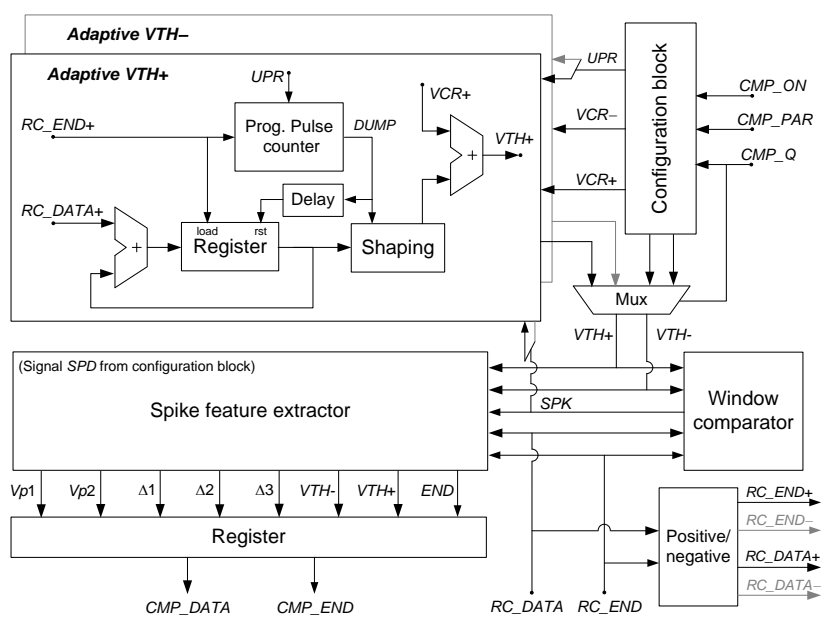

Figure 7. Structure of the data compression bock.

\section{Data compression block}

In this block, action potentials are detected by supervising the neural signal sequence $R C \_D A T A$ and identifying those instances which surpass the band comprised between a positive threshold $V_{T H}{ }^{+}$and a negative threshold $V T H^{-}$. Once an action potential is detected, the data compression block triggers a real-time process for extracting the parameters of a piecewise-linear (PWL) approximation of the spike in timevoltage domain. Such parameters, composed by voltage amplitude values and time intervals, define the set of parameters FE_DATA in Fig. 4.

Fig. 7 shows the structure of the data compression block. It comprises two structurally identical blocks, denoted as adaptive $V T H^{+}$and adaptive $V T H^{-}$, which operate respectively with the positive and negative values of $R C \_D A T A$ and allow the threshold voltages $V T H^{+}$and $V T H^{-}$to be dynamically adjusted to offset possible variations in the background noise of the captured signal. Additionally, it includes a window comparator for the detection of action potentials and a spike feature extractor, mainly composed of digital comparators and counters, for the characterization of the detected spikes. All the blocks are clocked by $C L K D$, excepting the spike feature extractor which operates at $C L K C$ rate. Moreover, as soon as the window comparator detects a spike, the clock of the ADC in the recording channel switches to $C L K C$. This is done to increase the resolution of the time parameters in set $F E_{-} D A T A$.

When the adaptive modules for $V T H^{+}$and $V T H^{-}$calculations are active (option $C M P \_Q$ is '001'), the standard deviations of background noise are obtained as

$$
\begin{aligned}
& \sigma^{+}(n)=\frac{1}{S} \sum_{n=1}^{S} R C_{-} D A T A^{+}(n) \\
& \sigma^{-}(n)=\frac{1}{S} \sum_{n=1}^{S} \text { RC_DAT } A^{-}(n)
\end{aligned}
$$

where $R C_{-} D A T A^{+}$and $R C_{-} D A T A^{-}$denote, respectively, the positive and negative values of $R C \_D A T A$, excluding those values belonging to detected spikes; $S=2^{U P R}$ and $U P R$ is a parameter which defines the refresh rate of the threshold voltages. Based on these estimations, $V T H^{+}$and $V T H^{-}$are calculated as:

$$
\begin{aligned}
& V T H^{+}=2 \sigma^{+}(n)+V C R^{+} \\
& V T H^{-}=2 \sigma^{-}(n)-V C R^{-}
\end{aligned}
$$

which are slightly different to the expressions given in [32] to allow for smoother variations of the threshold voltages with the background noise - one LSB variation in standard deviation gives rise to two LSBs corrections in threshold voltage. In (2), parameters $V C R^{+}$and $V C R^{-}$are user-defined correction terms for tweaking the threshold values.

As shown in Fig. 7, sums in (1) are implemented by accumulators and the number of additions is controlled by programmable pulse counters fed by end of conversion signals $R C \_E N D$. Every time the window comparator detects a spike, signal $S P K$ goes to high logic state and the counting operations in the adaptive $V T H^{+}$and adaptive $V T H^{-}$blocks are frozen. When any of the counts in these blocks reaches a value $S$, the corresponding pulse counter fires a signal DUMP which dumps the content of the register into a vector shaping block. After a clock cycle $C L K D$, the content of such register is emptied and the accumulation and counting process starts over. The shaping block shifts and truncates the dumped value so as to implement the scaling operation in (2) and, afterward, an adder sums up the values $V C R^{+}$or $V C R^{-}$, as corresponds.

The spike feature extractor identifies relevant coordinates of every spike waveform to compose the vector of representative values FE_DATA. These values are two voltage amplitude values, $V_{p 1}$ and $V_{p 2}$, and three time intervals, $\Delta_{1}, \Delta_{2}$ and $\Delta_{3}$. The time intervals are counted in CLKC clock cycles. With option $C M P \_Q$ is '001', the set FE_DATA also includes the value of the threshold voltage first crossed by the spike.

As shown in Fig. 8, the pair $\left(V_{p 1}, \Delta_{1}\right)$ corresponds to the position of the first voltage peak of the action potential. Value $\Delta_{1}$ is the time which elapses between threshold crossing and the instant when such peak is detected. Parameters $\left(V_{p 2}\right.$, $\Delta_{2}$ ) represent the position of the next peak when the neural potential shoots back in the opposite direction. In this case, $\Delta_{2}$ indicates the time which elapses between the two voltage peaks. Finally, $\Delta_{3}$ measures the time interval employed for returning to the resting level (mid code of the ADC range) from the second voltage peak. Value $\Delta_{3}$ is upper bounded by $S P D-\Delta_{1}-\Delta_{2}$, where parameter $S P D$ is the estimated duration of action potentials.

The proposed approach for spike detection based on dual adaptive threshold requires smaller computational resources than other more elaborate energy-based detectors [28] and does not add any extra delay on the process of identifying spikes [33], what paves the way for the real-time characterization of action potentials with no need to temporarily store previous records of the neural signal. Fig. 9(a) illustrates the average spike Detection Accuracy (DA) versus Signal to Noise Ratio (SNR) using the proposed technique. Following the procedures in [28] and [32], a total of 10 different neural segments (each with a different set of four identifiable action potentials) at 41 different noise levels (from -10 to $10 \mathrm{~dB}$ at $0.5 \mathrm{~dB}$ steps) were synthesized. The background noise was generated from 25 aggregated neural spikes with different firing rates. The duration of all the segments was 60s. As 


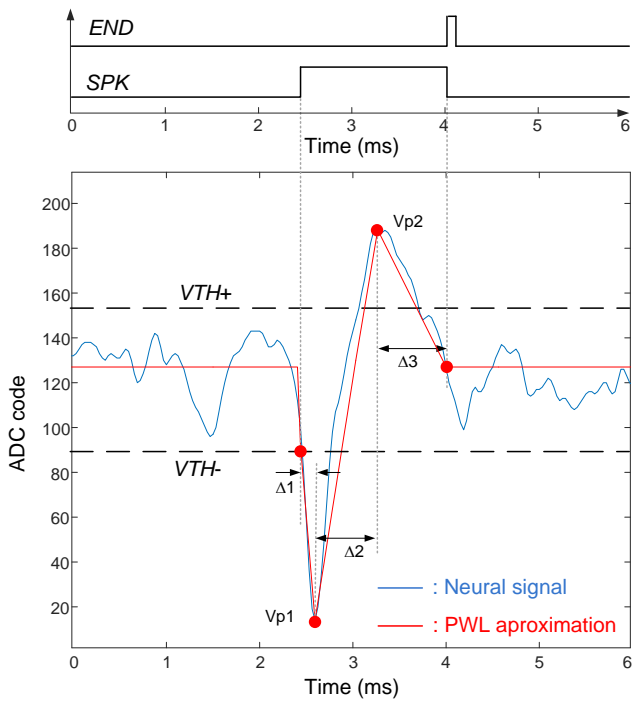

Figure 8. Piecewise-linear approximation of spikes.
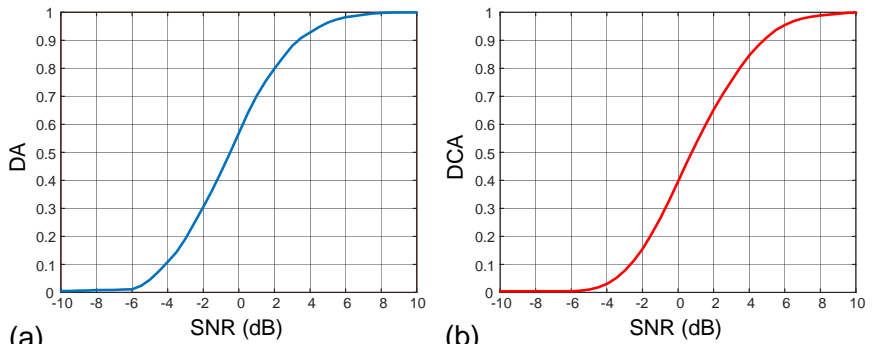

Figure 9. (a) Detection Accuracy (DA) and (b) Detection-Classification Accuracy (DCA) of the dual adaptive threshold for different values of SNR. Definitions for DA and DCA can be found in [34].

can be seen, the obtained DA is similar to that obtained in [34] (above $90 \%$ for a SNR of $5 \mathrm{~dB}$ ), thus confirming the improved performance of the dual threshold approach as compared to the median threshold technique [32]. However, while in [34] the threshold voltages are computed off-chip during a training phase, in the proposed approach they are calculated on-chip and they scale automatically with background noise. The average Detection-Classification Accuracy (DCA) measured over the same datasets is illustrated in Fig. 9(b). Spike classification (not implemented on-chip) has been made by $\mathrm{k}$-means clustering techniques using Euclidean distances [32]. Note that for SNRs above $5 \mathrm{~dB}$, the sorting error is below $10 \%$. These results are quite similar to those reported in [34] for the Zero-Crossing Feature (ZCF) and Principal Component Analysis (PCA) algorithms.

\section{ANALOG Front-END}

Fig. 10(a) shows the schematic of the analog front-end consisting of a bandpass programmable LNA and a gain adjustable ADC connected together by unity-gain buffers. The LNA uses two amplifier stages and local capacitive feedback around the first OTA. This architecture is used to create a double pole at the low-pass corner of the passband, thus leading to a $40 \mathrm{~dB} / \mathrm{dec}$ roll-off, which is beneficial for suppressing high frequency noise components in the captured signal. The midband gain of the structure is given by the ratio between the input and the feedback capacitance, $C_{i} / C_{f}$ and the position of the high-pass and low-pass corners are approximately given by $1 / R_{f} C_{f}$ and $2 g_{m 2} C_{f} / C_{c} C_{l}$, respectively. The first OTA $\left[\mathrm{OTA}_{1}\right.$, illustrated in Fig. 10(b)] uses a complementary input differential pair to nearly double its equivalent transconductance for the same biasing current. The second OTA $\left[\mathrm{OTA}_{2}\right.$, illustrated in Fig. 10(c)] uses a simple $p$-input class-A stage. Each feedback resistor, $R_{f}$, is implemented as a 3-bit $(N H P=3)$ digitally-controlled tapped cascade of transistors biased in deep subthreshold region [35]. The range of $R_{f}$ values sweep from 5.5 to $85 \mathrm{GOhm}$. This allows to externally tune the position of the HP corner from 15 to $232 \mathrm{~Hz}$. Similarly, a 2-bit $(N L P=2)$ programmable capacitive bank, $C_{l}$, loading the structure allows the tuning of the LP corner between $5.2 \mathrm{kHz}$ and $10.15 \mathrm{kHz}$. These ranges clearly cover the frequency band of action potentials and partially cover the band of local field potentials ( $\beta$ band and above).

The switched capacitor (SC) structure at the right hand side of Fig. 10(a) implements the ADC of the recording channel [36]. It is built around a SC integrator whose gain can be controlled from 0 to $18 \mathrm{~dB}$ with 3-bit $(N P G=3)$ programmable input capacitor banks, $C_{i n}$. Hence, besides conversion, the circuit features PGA operation. The OTA of the integrator $\left[\mathrm{OTA}_{3}\right.$, illustrated in Fig. 10(d)] uses an $n$ input folded-cascode stage and the comparator employs a current-controlled dynamic latch. Outputs bits are derived by successively detecting the sign of the voltage stored in the integrator. The timing diagram is shown in Fig. 10(e). Depending on the output of the comparator, the integrated voltage is updated by adding or subtracting binary scaled versions of a voltage reference $V_{\text {ref }}$. These voltages $V_{j}=V_{\text {ref }} / 2^{j}$, $j=1, \ldots, n-1$, where $n$ is the output resolution of the converter, are obtained by capacitive voltage division at every step of the conversion process. Solved bits are stored in a SAR register. In the presented design, the bias current $\mathrm{OTA}_{3}$ is dynamically adapted for power saving by taking advantage that settling requirements are progressively relaxed along the conversion.

Full details on the analysis, design and characterization of the LNA and the programmable gain ADC are given in [37] and [36], respectively. Herein, for the sake of completeness, only the performance summary of both blocks is given in Table VI. The power consumption of the LNA including biasing and common-mode feedback circuits, as well as, output buffers, is $1.92 \mu \mathrm{W}$, no matter the SoC operation mode. The power dissipation of the PGA/ADC, when operated at $C L K D$, is $0.51 \mu \mathrm{W}$ for a sampling rate of about $30 \mathrm{kS} / \mathrm{s}$. During the extraction of spike features, the clock changes to CLKC and the sampling rate raises to about $90 \mathrm{kS} / \mathrm{s}$. In this case, the power consumption of the PGA/ADC is $1.52 \mu \mathrm{W}$.

\section{EXPERIMENTAL RESUlTS}

Fig. 11(a) shows the microphotograph of SoC fabricated in a standard $0.13 \mu \mathrm{m}$ CMOS process. It occupies an active 


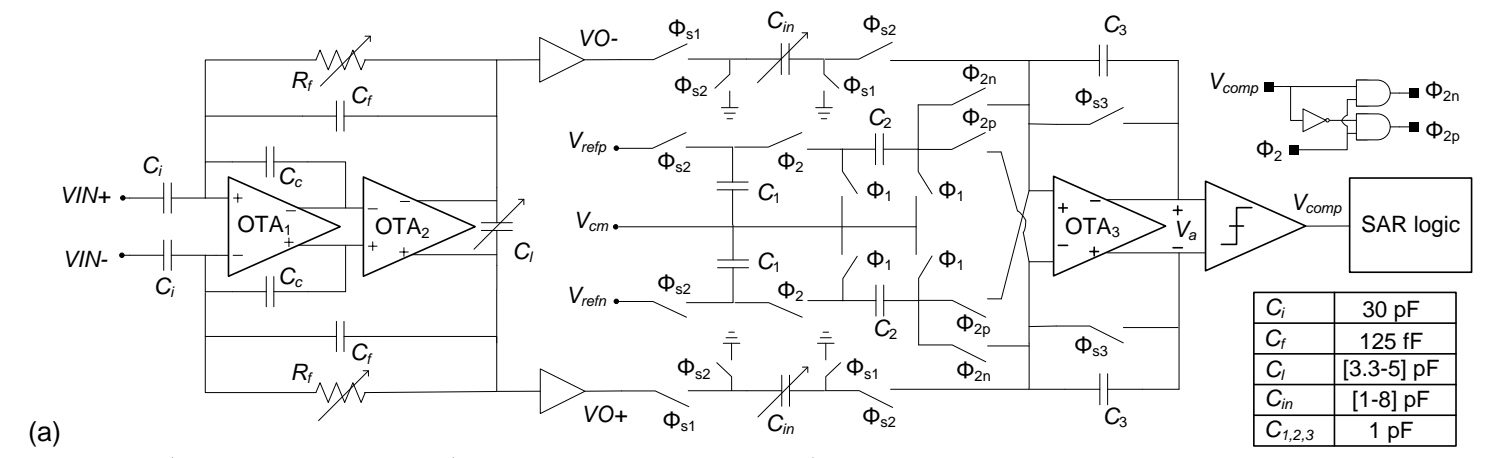

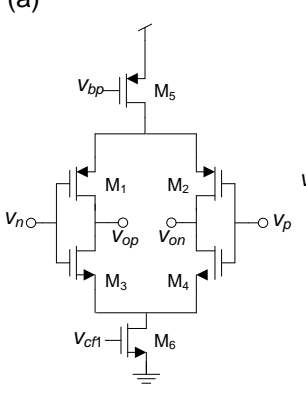

(b)

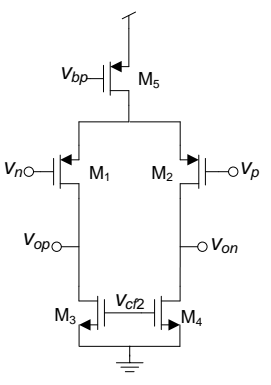

(c)

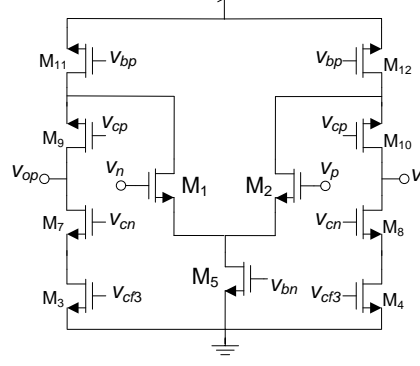

(d)

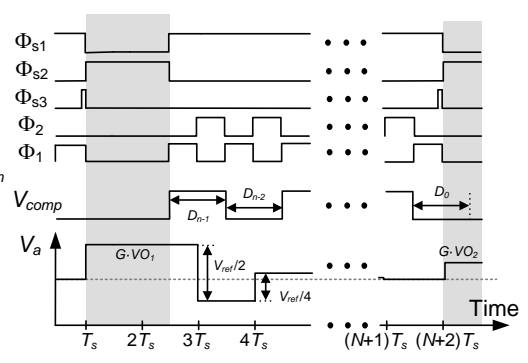

(e)

Figure 10. (a) Schematic of the analog front-end. Capacitor sizes are shown in the table. (b-d) Schematics of OTA 1 , OTA 2 and OTA 3 , respectively. (e) Timing diagram of the programmable gain ADC.

Table I

PERFormanCE SUMMARY OF THE LNA AND THE PROGRAMMABLE GAIN ADC.

\begin{tabular}{|c|c|}
\hline Technology & $0.13 \mu \mathrm{m} 6 \mathrm{M} 2 \mathrm{P}$ CMOS \\
\hline Supply voltage & $1.2 \mathrm{~V}$ \\
\hline Area/channel & $0.16 \mathrm{~mm}^{2}$ \\
\hline \multicolumn{2}{|l|}{ Low Noise Amplifier } \\
\hline \multicolumn{2}{|l|}{\begin{tabular}{|l|l} 
& High pass freq. $(\mathrm{Hz})$
\end{tabular}} \\
\hline Low pass freq. $(\mathrm{kHz})$ & $5.2-10.15$ \\
\hline Input ref. noise $\left(\mu \mathrm{V}_{\mathrm{rms}}\right)$ & 3.8 \\
\hline Noise int. bandwidth $(\mathrm{Hz})$ & $1-100 \mathrm{k}$ \\
\hline Bandwidth (Hz) & $192-7.4 \mathrm{k}$ \\
\hline Gain $(\mathrm{dB})$ & 46 \\
\hline Input impedance @ 1 kHz (MOhm) & 5.4 \\
\hline CMRR / PSRR (dB) & $85 / 75$ \\
\hline THD & $0.08 \% @ 3.0 \mathrm{mV}_{\mathrm{pp}}$ \\
\hline Power cons. $(\mu \mathrm{W})$ & 1.92 \\
\hline$N E F$ & 2.16 \\
\hline$N E F^{2} \cdot \mathrm{V}_{\mathrm{DD}}$ & 5.59 \\
\hline \multicolumn{2}{|l|}{ Programmable-gain ADC } \\
\hline Gain range $(\mathrm{dB})$ & $0-18$ \\
\hline Input range & $1.2 \mathrm{Vpp}$ \\
\hline INL (LSB) & $-0.4-0.5$ \\
\hline DNL (LSB) & $-0.25-0.48$ \\
\hline Sampling rate $(\mathrm{kS} / \mathrm{s})$ & 90 \\
\hline ENOB & 7.60 \\
\hline Power cons. $(\mu \mathrm{W})$ & 0.51 \\
\hline
\end{tabular}

area of $13.45 \mathrm{~mm}^{2}$ with each channel having $400 \mu \mathrm{m}$ pitch - see channel floor-plan in Fig. 11(b). DCOs are placed on the left side of the recording array, while the rest of blocks (communications node, power management, RF transceiver, and some test circuits) are placed to the right. The communications node is on deep n-well for digital noise isolation. Besides an external padring, each recording channel embeds a small pad for flip-chiping. These pads include ESD protection diodes which are connected to a voltage clamp protection

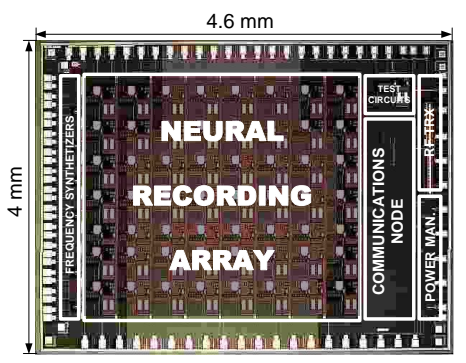

(a)

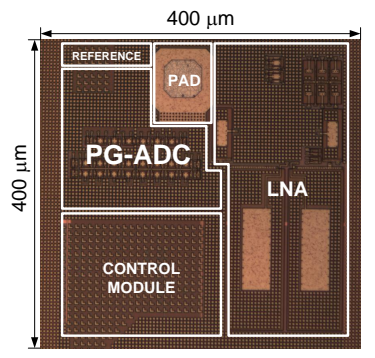

(b)
Figure 11. Microphotograph of the (a) 64-channel neural sensor, (b) single channel.

ring surrounding the array. Analog references (common-mode voltage, ADC voltage references and current bias references) for the recording channels are shared by columns. Digital signals crossing the array are distributed in eight output branches (one per row).

\section{A. Benchtop characterization}

Measurements of the isolated analog building blocks are reported elsewhere [37], [36]. In this section focus is paid on the characterization of the control module and, in particular, on the functional assessment of the Calibration and Data Compression modes. Neural signal segments captured in vivo (see Sec. VII-B2) and resynthesized in the laboratory by means of a TT $i$ TGA12101 arbitrary function generator have been used in the following experiments.

Figure 12 shows the measured ADC output code, as well as the evolution of the control words $H P C$ and $L P C$, along the calibration of the channel bandpass characteristic. Seeking for neural spike recordings, the target frequencies for the HP and 


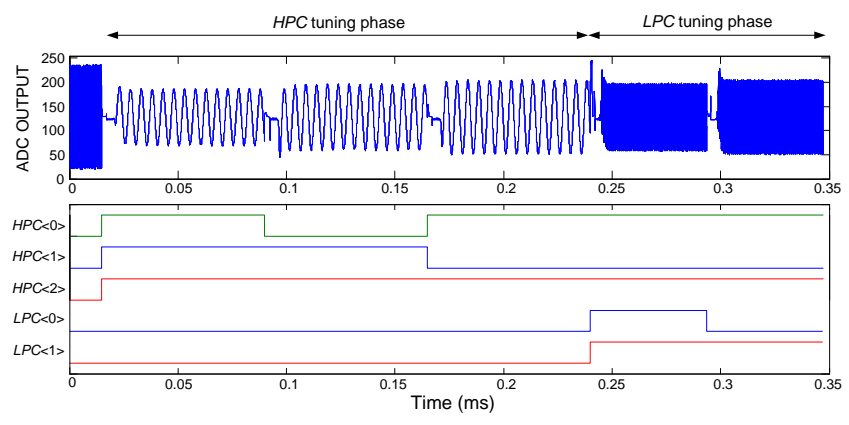

Figure 12. Evolution during BP-LNA calibration.
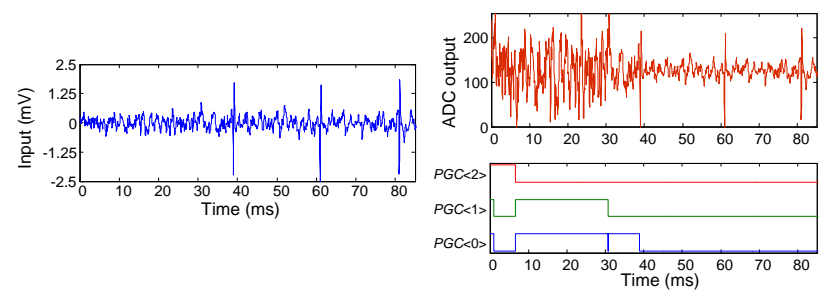

Figure 13. Evolution during PGA calibration.

LP poles are $200 \mathrm{~Hz}$ and $7 \mathrm{kHz}$, respectively. As can be seen, three iterations are needed to identify the code $(H P C=$ ' 101 ') that better approximates the desired HP pole, whereas two iterations suffice for the LP pole $(L P C=$ ' 10 ').

Figure 13 shows the measured ADC output code and $P G C$ control word along the calibration of the PGA (BP_CAL is set to ' 0 '). The input voltage provided by the function generator was set to a large value of $5 \mathrm{mV}_{\mathrm{pp}}$. As can be seen, the control module of the recording channel initially sets the PGA to its maximum gain value $(18 \mathrm{~dB})$ and the implemented algorithm decreases $P G C$ every time a saturation is detected at the output of the ADC. At the end of the adjustment process, the $P G C$ output code is '000' as expected.

Fig. 14 illustrates the operation of the adaptive threshold algorithm implemented in the local digital processor of the channels. At the system start-up, the positive and negative threshold voltages are set to the upper and lower bounds of the ADC code, respectively, and, after a transient, these voltages settle to the correct values. In this experiment, $U P R=9$, $V C R^{+}=6$ and $V C R^{-}=6$. It is worth observing that the positive and negative threshold levels self-adapt to changes in the background noise, particularly, when bursts of spikes occur.

Fig. 15 illustrates the operation of a neural channel in the Data Compression mode. The SPD parameter was set to $1.75 \mathrm{~ms}$. If no spike is detected, the data compression block of the recording channel sends no information. When a spike is detected, the spike feature extractor obtains the parameters of the PWL representation (in the figure, the characterization of the three detected spikes are given in corresponding tables). Regarding the reconstruction accuracy of the compression, we have reproduced in the laboratory different experimental neural sequences, taken from different channels and recording sessions under mode $T R \_Q=$ ' 000 ', totalizing some 25.000 detected spikes. The root-mean-square deviation between the

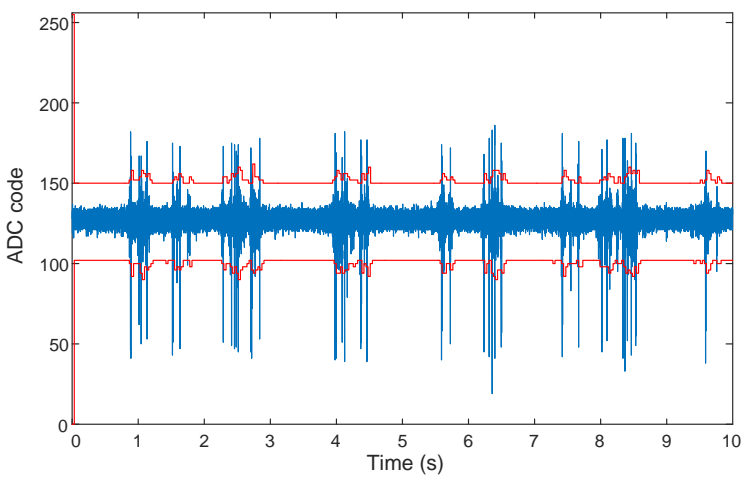

Figure 14. Adaptive threshold voltage algorithm: Neural signal (blue), threshold voltages (red).

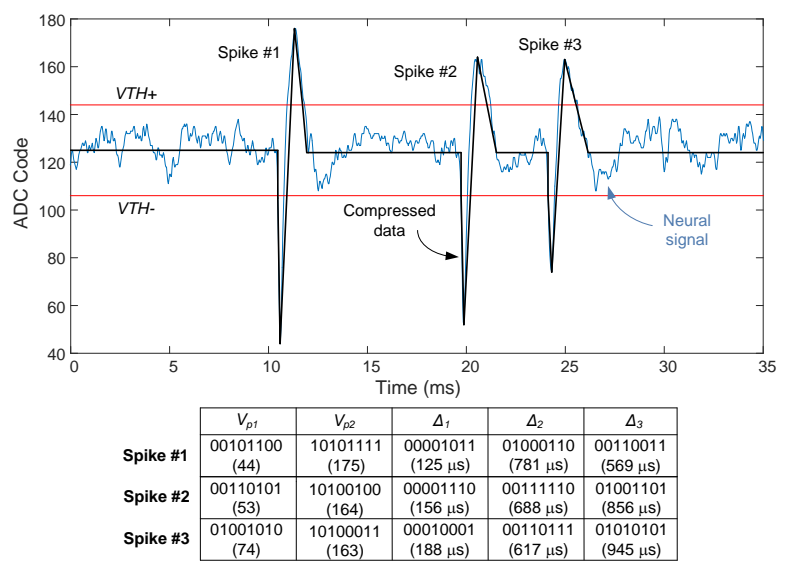

Figure 15. Measurement under Data Compression mode.

original waveforms and the generated PWL representations amounts $8.25 \pm 2.68 \%$.

In the Signal Monitoring mode, the power consumption of the SoC, excluding the power management unit and the wireless transceiver, raises with the number of active channels: $69.03 \mu \mathrm{W}$ (one active column, $T R \_Q==^{\prime} 000$ '), $98.64 \mu \mathrm{W}$ (two consecutive active columns, $T R_{-} Q==^{\prime} 001$ '), $142.28 \mu \mathrm{W}$ (four consecutive active columns, $T R_{-} Q={ }^{\prime} 010^{\prime}$ ), $241.56 \mu \mathrm{W}$ (whole array, $\left.T R \_Q=' 011^{\prime}\right)$. In all these configurations, however, the power consumption of the communications node keeps fairly to the same value (about $47 \mu \mathrm{W}$ ) because active channels and throughput rate are traded off. The most demanding operation mode is the Data Compression mode with adaptive thresholding enabled for which the power consumption is $330 \mu \mathrm{W}$. This is mainly because the power consumption per channel raises from $3.04 \mu \mathrm{W}$ (in Signal Monitoring mode) to $4.54 \mu \mathrm{W}$.

\section{B. In vivo Characterization}

1) Measurement setup: Fig. 16 shows the schematic diagram of the measurement setup used for in vivo neural recording. This setup is not intended for mesurements on freely moving animals but for validating and quantifying the SoC performance with two different types of microelectrode arrays (MEAs). In one case, a flexible sub-dural microelectrode array (Multi Channel Systems MCS GmbH) with TiN 


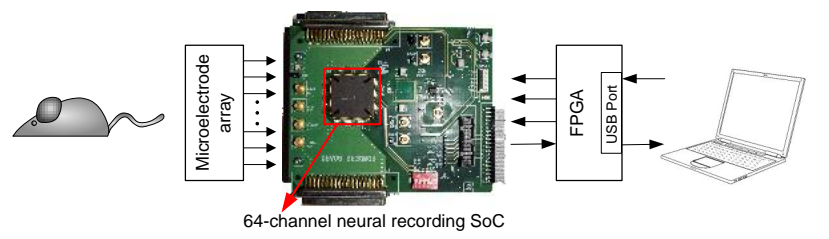

Figure 16. Measurement setup and PCB of the SoC.

(a)
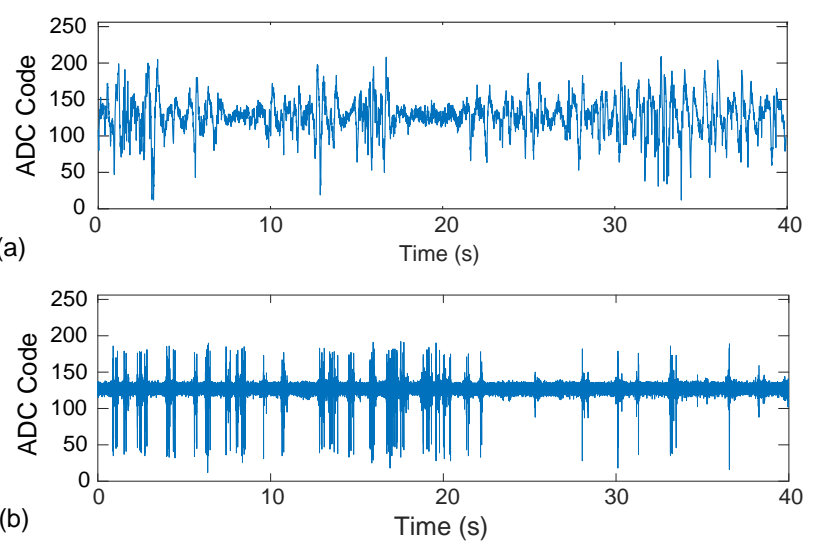

Figure 17. (a) LFP activity recorded in vivo by using a flexible sub-dural array. (b) Neural spike activity recorded in vivo by using a Utah array.

electrodes separated by $300 \mu \mathrm{m}$ (diameter $30 \mu \mathrm{m}$ ) placed on a polymide substrate was used. The signals captured by the microelectrodes were transferred to the 64-channel neural recording system by means of flat ribbon cables connected between the MEA adapter (ADPT-FM-36, MCS GmbH) and the SoC through row precision sockets from Samtec. In the second case, the MEA was a Utah array with an ICS-96 connector (Blackrock Microsystems LLC). A custom adapter was designed for arranging the pin distributions of the ICS-96 connector (only, banks A and C) so that the same connection strategy employed with the flexible MEA could be reused.

A Field Programmable Gate Array (Nexys ${ }^{\mathrm{TM}} 2$ Spartan-3E FPGA Board by Digilent) was used to communicate the 64channel neural recording SoC with a host computer through a conventional 2.0 USB port. A user interface developed in $\mathrm{C}++$ was designed to control the whole measurement setup. The communications between the SoC and the FPGA only need one downward connection for the transfer of commands, and one upward connection for the reception of the neural information recorded and processed by the SoC. Additionally, a $4 \mathrm{MHz}$ master clock is transferred for timing purposes. Details on the FPGA programming were given in [38].

All experimental procedures were performed in conformance to the directive 2010/63/EU of the European Parliament and of the Council, and the RD 53/2013 Spanish regulation on the protection of animals use for scientific purposes and approved by the Miguel Hernandez University Committee for Animal use in Laboratory. Animal models were adult male Long Evans rats.

2) In vivo results: Fig. 17(a) shows a 40 s neural recording segment captured from one of the channels of the SoC when operated in Signal Monitoring mode with the column scanning option enabled $(T R Q=$ '011', 64 channels tracked at a throughput rate per channel of $3.5 \mathrm{kS} / \mathrm{s}$ ). The flexible sub-dural microelectrode array described in Sec. VII-B1 was used in this experiment. Although the experiment lasted for several hours, no substantial differences were observed in the course of the measurement, nor among the channels. In this experiment, all the 64 channels of the array were selected and their bandpass characteristics were set between $15 \mathrm{~Hz}$ and $5.2 \mathrm{kHz}$. The control word of the PGA was set to $P G C=$ ' 101 '.

Fig. 17(b) shows the recording captured from one of the channels of the SoC when operated in Signal Monitoring mode from two columns of the channel array $(T R Q=$ '001', 16 channels tracked at a throughput rate per channel of $15 \mathrm{kS} / \mathrm{s}$ ). In this case, the Utah array was employed in the in vivo measurement. The bandpass characteristics of the 16 selected channels were set between $200 \mathrm{~Hz}$ and $7 \mathrm{kHz}$ in order to capture spike activity. The $P G C$ word was set to '111'. As can be observed in Fig. 17(b), isolated action potentials and bursts of spikes are clearly noticeable in the recording. As in the previous case, no substantial difference from the shown performance was observed along the experiment nor among the channels.

Fig. 18 illustrates the system operation in the Data Compression mode using the Utah array, as well as, the PWL approximation of the signal captured by one of the channels. Dots represent the spikes detected by the neural array in a time slot of $5 \mathrm{~s}$. Adaptive threshold calculation was enabled and, as before, the $S P D$ parameter was set to $1.75 \mathrm{~ms}$. The main digital processor cyclically examines the routers and transmits their contents when ready. The system requires $22 \mu$ s to transmit the information of one spike. This is much lower than a typical spike duration and, of course, much lower than the time basis for firing occurrences. It means, that no information is lost not even in the unlikely case all the channels fire at the same instant.

\section{State of the Art comparison}

Table II compares the proposal to the state-of-the-art. The power consumption of the transceiver and the power management unit in those solutions which include wireless data transmission and remote powering has not been computed in the total power. Similarly, if the reported chip includes stimulation circuitry, its consumption has not been accounted for either. It is worth observing that the proposed solution is one of the most power efficient recording systems, in spite it offers external programming capabilities not covered by others.

\section{CONCLUSIONS}

A 64-channel reconfigurable, self-calibrated neural recording/communication SoC with embedded data reduction techniques fabricated in a standard CMOS $130 \mathrm{~nm}$ process is reported. Each channel embeds all the circuitry to filter, amplify and digitizes the input data, as well as compress the detected neural spike activity, minimizing the amount of generated data. A distributed digital signal processing approach, with 
Table II

PERFORMANCE SUMMARY AND COMPARISON

\begin{tabular}{|c|c|c|c|c|c|c|c|c|c|c|}
\hline & {$[26]$} & [15] & [39] & [24] & [40] & [41] & [18] & [27] & [25] & This work \\
\hline Technology (nm) & 180 & 350 & 180 & 350 & 130 & 180 & 180 & 65 & 65 & 130 \\
\hline Supply voltage (V) & 1.8 & 3.3 & $1.0 / 1.8$ & 1.5 & $1.2 / 3.3$ & $1.0 / 1.8$ & 0.45 & 0.5 & $1.0 / 0.8$ & 1.2 \\
\hline Number of channels & 16 & 128 & 32 & 8 & 64 & 100 & 100 & 64 & 64 & 64 \\
\hline Total power $(\mu \mathrm{W})$ & 680 & 4400 & 325 & 375 & 640 & 1160 & 94 & 147.2 & 229.4 & $<330$ \\
\hline Power / channel $(\mu \mathrm{W})$ & 42.5 & 62.9 & 10.1 & 26.9 & 10 & 11.6 & 0.94 & 2.3 & 3.5 & $3.04 / 4.54$ \\
\hline Max. system gain (dB) & 70 & 60 & 66 & 65.5 & 60 & 60.9 & 55.5 & N.A. & 65 & 63 \\
\hline High pass freq. $(\mathrm{Hz})$ & 100 & $0.1-200$ & 350 & $1.1-525$ & 1 & $0.38-248$ & 0.25 & $1-8$ & $3-8$ & $15-232$ \\
\hline Low pass freq. $(\mathrm{kHz})$ & 9.2 & $2-20$ & 12.0 & $5.1-12$ & 5 & $0.432-5.1$ & $2.5-10$ & 0.4 & $10-1000$ & $5.2-10.15$ \\
\hline Input ref. noise $\left(\mu V_{r m s}\right)$ & 5.4 & 4.9 & 5.4 & 3.12 & 5.1 & 4 & 3.2 & 1.27 & 7.5 & 3.8 \\
\hline NEF & 4.9 & - & 4.4 & 2.68 & 4.4 & 1.9 & 1.57 & 4.76 & 3.6 & 2.16 \\
\hline ENOB (bit) & 7.0 & $6-9$ & 7.65 & 9.1 & 7.6 & 8.3 & 8.27 & 15 & 8.2 & 7.65 \\
\hline Samp. freq. / channel $(\mathrm{kS} / \mathrm{s})$ & 30 & 40 & 31.25 & 63 & 12.5 & 24.5 & 20 & 1 & 20 & $30 / 90$ \\
\hline Data bitrate reduction & Yes & Yes & No & Yes & Yes & No & No & No & Yes & Yes \\
\hline Self-calibration & No & No & No & No & No & No & No & No & No & Yes \\
\hline Adaptive threshold & No & Yes & No & No & No & No & No & No & Yes & Yes \\
\hline Stimulation & No & No & No & Yes & Yes & No & No & No & Yes & No \\
\hline Wireless data/power & No & Yes & No & Yes & Yes & No & No & Yes & No & Yes \\
\hline
\end{tabular}
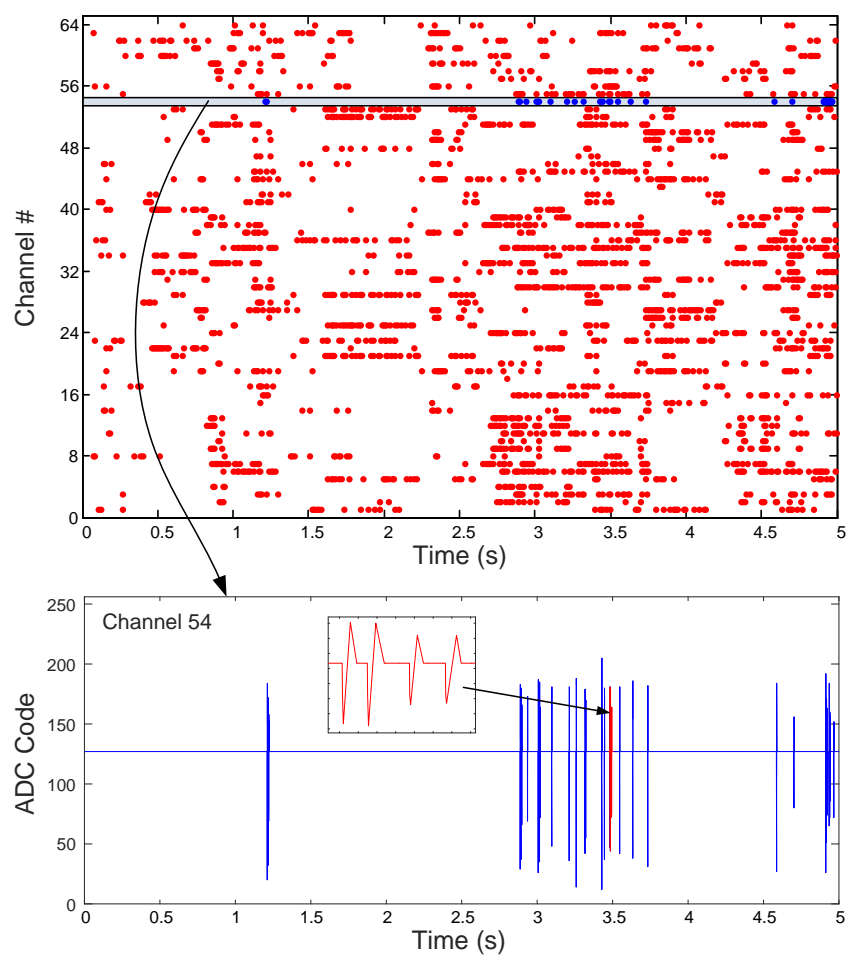

Figure 18. Data output stream under Data Compression mode.

tasks at channel- and array levels, has been found an efficient solution for reducing the power consumption of the $\mathrm{SoC}$ and simplifying communications through the array.

The communications section of the SoC defines the operation mode of the recording channels and implements a fullduplex communication protocol for data transmission through the wireless link. In one operation mode, the selected channels can be configured to detect and compress neural spikes so that feature vectors instead of raw signal samples are transferred. In another mode, the system implements a self-calibration algorithm which automatically adapts the filter bandwidth and the gain setting of the channels. The system also offers different alternatives for raw data transmission in which the number of active channels and the effective sampling rate are traded off. In all cases, the total throughput rate of the SoC keeps below 4.0 Mbps as imposed by the wireless link. The sensor consumes $330 \mu \mathrm{W}$ from a $1.2 \mathrm{~V}$ voltage supply in the Data Compression mode, the most demanding one.

\section{REFERENCES}

[1] D. Borton, M. Yin, J. Aceros, and A. Nurmikko, "An implantable wireless neural interface for recording cortical circuit dynamics in moving primates," Journal of Neural Engineering, vol. 10, no. 2, p. 026010, Apr. 2013.

[2] A. Berényi, Z. Somogyvári, A. Nagy, L. Roux, J. Long, S. Fujisawa, E. Stark, A. Leonardo, T. Harris, and G. Buzsáki, "Large-scale, highdensity (up to 512 channels) recording of local circuits in behaving animals," Journal of Neurophysiology, vol. 111, no. 5, pp. 1132-1149, Mar. 2014.

[3] G. Charvet et al., "Biomea: A versatile high-density 3d microelectrode array system using integrated electronics," Biosensors and Bioelectronics, vol. 25, no. 8, pp. 1889-1896, Apr. 2010.

[4] M. Sawan, M. Salam, J. Le Lan, A. Kassab, S. Gelinas, P. Vannasing, F. Lesage, M. Lassonde, and D. Nguyen, "Wireless recording systems: From noninvasive eeg-nirs to invasive eeg devices," Biomedical Circuits and Systems, IEEE Transactions on, vol. 7, no. 2, pp. 186-195, 2013.

[5] W. Truccolo, J. A. Donoghue, L. R. Hochberg, E. N. Eskandar, J. R. Madsen, W. S. Anderson, E. N. Brown, E. Halgren, and S. S. Cash, "Single-neuron dynamics in human focal epilepsy," Nature Neuroscience, vol. 14, no. 5, pp. 635-641, May 2011.

[6] E. Tóth, D. F. Fabó, L. Entz, I. Ulbert, and L. Eróss, "Intracranial neuronal ensemble recordings and analysis in epilepsy," Journal of Neuroscience Methods, vol. 260, pp. 261-269, Feb. 2016.

[7] B. Rosin, M. Slovik, R. Mitelman, M. Rivlin-Etzion, S. Haber, Z. Israel, E. Vaadia, and H. Bergman, "Closed-loop deep brain stimulation is superior in ameliorating parkinsonism," Neuron, vol. 72 , no. 2, pp. 370384, Oct. 2011.

[8] S. A. Deadwyler, R. E. Hampson, D. Song, I. Opris, G. A. Gerhardt, V. Z. Marmarelis, and T. W. Berger, "A cognitive prosthesis for memory facilitation by closed-loop functional ensemble stimulation of hippocampal neurons in primate brain," Experimental Neurology, 2016.

[9] A. Schwartz, X. Cui, D. Weber, and D. Moran, "Brain-controlled interfaces: Movement restoration with neural prosthetics," Neuron, vol. 52, pp. 205-220, 2006.

[10] L. Hochberg et al., "Reach and grasp by people with tetraplegia using a neurally controlled robotic arm," Nature, vol. 485, no. 7398, pp. 372375, May 2012.

[11] S. Rajangam, P.-H. Tseng, A. Yin, G. Lehew, D. Schwarz, M. A. Lebedev, and M. A. L. Nicolelis, "Wireless Cortical Brain-Machine Interface for Whole-Body Navigation in Primates," Nature Scientific Reports, vol. 6, Mar. 2016. 
[12] K. Abdelhalim, L. Kokarovtseva, J. Pérez-Velazquez, and R. Genov, "915-mhz fsk/ook wireless neural recording soc with 64 mixed-signal fir filters," Solid-State Circuits, IEEE Journal of, vol. 48, no. 10, pp. 2478-2493, Oct. 2013.

[13] J. Aziz, K. Abdelhalim, R. Shulyzki, R. Genov, B. Bardakjian, M. Derchansky, D. Serletis, and P. L. Carlen, "256-channel neural recording and delta compression microsystem with $3 \mathrm{~d}$ electrodes," Solid-State Circuits, IEEE Journal of, vol. 44, pp. 995-1005, 2009.

[14] W. Biederman, D. Yeager, N. Narevsky, A. Koralek, J. Carmena, E. Alon, and J. Rabaey, "A fully-integrated, miniaturized $0.125 \mathrm{~mm} 2 ; 10.5$ uw wireless neural sensor," Solid-State Circuits, IEEE Journal of, vol. 48, no. 4, pp. 960-970, Apr. 2013.

[15] M.-S. Chae, Z. Yang, M. Yuce, L. Hoang, and W. Liu, "A 128-channel $6 \mathrm{mw}$ wireless neural recording ic with spike feature extraction and uwb transmitter," Neural Systems and Rehabilitation Engineering, IEEE Transactions on, vol. 17, no. 4, pp. 312-321, 2009.

[16] A. Borna and K. Najafi, "A low power light weight wireless multichannel microsystem for reliable neural recording," Solid-State Circuits, IEEE Journal of, vol. 49, no. 2, pp. 439-451, Feb. 2014.

[17] H. Gao, R. Walker, P. Nuyujukian, K. Makinwa, K. Shenoy, B. Murmann, and T. Meng, "Hermese: A 96-channel full data rate direct neural interface in 0.13 um cmos," Solid-State Circuits, IEEE Journal of vol. 47, no. 4, pp. 1043-1055, 2012.

[18] D. Han, Y. Zheng, R. Rajkumar, G. Dawe, and M. Je, "A 0.45 v 100channel neural-recording ic with sub-uw/channel consumption in 0.18 um cmos," Biomedical Circuits and Systems, IEEE Transactions on, vol. 7, no. 6, pp. 735-746, Dec. 2013

[19] S.-B. Lee, L. H.-M., M. Kiani, U.-M. Jow, and M. Ghovanloo, "An inductively powered scalable 32-channel wireless neural recording system-on-a-chip for neuroscience applications," Biomedical Circuits and Systems, IEEE Transactions on, vol. 4, no. 6, pp. 360-371, 2010.

[20] R. Shulyzki, K. Abdelhalim, A. Bagheri, M. Salam, C. Florez, J. PérezVelazquez, P. Carlen, and R. Genov, "320-channel active probe for highresolution neuromonitoring and responsive neurostimulation," Biomedical Circuits and Systems, IEEE Transactions on, vol. 9, no. 1, pp. 34-49, Feb. 2015.

[21] A. M. Sodagar, G. E. Perlin, Y. Ying, K. Najafi, and K. D. Wise, "An implantable 64-channel wireless microsystem for single-unit neural recording," Solid-State Circuits, IEEE Journal of, vol. 44, no. 9, pp. 2591-2604, 2009

[22] X. Zou et al., "A 100-channel 1-mw implantable neural recording ic," Circuits and Systems I: Regular Papers, IEEE Transactions on, vol. 60, no. 10, pp. 2584-2596, Oct. 2013.

[23] R. Harrison, P. Watkins, R. Kier, R. Lovejoy, D. Black, B. Greger, and F. Solzbacher, "A low-power integrated circuit for a wireless 100electrode neural recording system," Solid-State Circuits, IEEE Journal of, vol. 42, no. 1, pp. 123-133, 2007.

[24] M. Azin, D. Guggenmos, S. Barbay, R. Nudo, and P. Mohseni, "A battery-powered activity-dependent intracortical microstimulation ic for brain-machine-brain interface," Solid-State Circuits, IEEE Journal of, vol. 46, no. 4, pp. 731-745, 2011.

[25] W. Biederman, D. Yeager, N. Narevsky, J. Leverett, R. Neely, J. Carmena, E. Alon, and J. Rabaey, "A $4.78 \mathrm{~mm} 2$ fully-integrated neuromodulation soc combining 64 acquisition channels with digital compression and simultaneous dual stimulation," Solid-State Circuits, IEEE Journal of, vol. 50, no. 4, pp. 1038-1047, Apr. 2015.

[26] B. Gosselin, A. Ayoub, J. Roy, M. Sawan, F. Lepore, A. Chaudhuri, and D. Guitton, "A mixed-signal multichip neural recording interface with bandwidth reduction," Biomedical Circuits and Systems, IEEE Transactions on, vol. 3, no. 3, pp. 129-141, 2009.

[27] R. Muller et al., "A minimally invasive 64-channel wireless uecog implant," Solid-State Circuits, IEEE Journal of, vol. 50, no. 1, pp. 344 359, Jan. 2015.

[28] S. Gibson, J. Judy, and D. Markovic, "Technology-aware algorithm design for neural spike detection, feature extraction, and dimensionality reduction," Neural Systems and Rehabilitation Engineering, IEEE Transactions on, vol. 18, pp. 469-478, 2010.

[29] A. Rodríguez-Pérez, M. Delgado-Restituto, A. Darie, C. Soto-Sánchez, E. Fernández-Jover, and A. Rodríguez-Vázquez, "A 330 uw, 64-channel neural recording sensor with embedded spike feature extraction and autocalibration," in Solid-State Circuits Conference (A-SSCC), 2014 IEEE Asian, Nov. 2014, pp. 205-208.

[30] K. Finkenzeller, RFID Handbook: Fundamentals and Applications in Contactless Smart Cards, Radio Frequency Identification and NearField Communication, 3rd Edition. Wiltshire, UK: Wiley, 2010. [Online]. Available: http://eu.wiley.com/WileyCDA/WileyTitle/productCd0470695064.html
[31] A. Rodríguez-Pérez, M. Delgado-Restituto, and A. Rodríguez-Vázquez, "Self-calibration of neural recording sensors," in 2014 IEEE Biomedical Circuits and Systems Conference (BioCAS), Oct. 2014, pp. 663-666.

[32] R. Quiroga, Z. Nadasdy, and Y. Ben-Shaul, "Unsupervised spike detection and sorting wavelets and superparamagnetic clustering," Neural Computation, vol. 16, pp. 1661-1687, 2004. [Online]. Available: http://www2.le.ac.uk/centres/csn/research-2/spike-sorting

[33] I. Obeid and P. Wolf, "Evaluation of spike-detection algorithms fora brain-machine interface application," IEEE Transactions on Biomedical Engineering, vol. 51, no. 6, pp. 905-911, 2004.

[34] A. Kamboh and A. Mason, "Computationally Efficient Neural Feature Extraction for Spike Sorting in Implantable High-Density Recording Systems," IEEE Transactions on Neural Systems and Rehabilitation Engineering, vol. 21, no. 1, pp. 1-9, Jan. 2013.

[35] A. Rodríguez-Pérez, J. Ruiz-Amaya, M. Delgado-Restituto, and A. Rodríguez-Vázquez, "A low-power programmable neural spike detection channel with embedded calibration and data compression," Biomedical Circuits and Systems, IEEE Transactions on, vol. 6, no. 2, pp. 87-100, 2012.

[36] A. Rodríguez-Pérez, M. Delgado-Restituto, and F. Medeiro, “A 515 nw, 0-18 db programmable gain analog-to-digital converter for in-channel neural recording interfaces," Biomedical Circuits and Systems, IEEE Transactions on, vol. 8, no. 3, pp. 358-370, 2014.

[37] J. Ruiz-Amaya, A. Rodríguez-Pérez, and M. Delgado-Restituto, "A low noise amplifier for neural spike recording interfaces," Sensors (MDPI), vol. 15 , no. 10 , pp. 25313-25335, Sep. 2015. [Online]. Available: http://www.mdpi.com/1424-8220/15/10/25313

[38] M. Delgado-Restituto, A. Rodríguez-Pérez, A. Darie, A. RodríguezVázquez, C. Soto-Sánchez, and E. Fernández-Jover, "In vivo measurements with a 64-channel extracellular neural recording integrated circuit," in 2014 21st IEEE International Conference on Electronics, Circuits and Systems (ICECS), Dec. 2014, pp. 486-489.

[39] W. Wattanapanitch and R. Sarpeshkar, "A low-power 32-channel digitally programmable neural recording integrated circuit," IEEE Trans. on Biom. Circ. and Systems, vol. 5, no. 6, pp. 592-602, 2011.

[40] K. Abdelhalim, H. Jafari, L. Kokarovtseva, J. Perez Velazquez, and R. Genov, "64-channel uwb wireless neural vector analyzer soc with a closed-loop phase synchrony-triggered neurostimulator," IEEE Journal of Solid-State Circuits, vol. 48, no. 10, pp. 2494-2510, Oct. 2013.

[41] X. Zou et al., "A 100-channel 1-mW implantable neural recording IC," IEEE Trans. on Circ. and Systems I, vol. 60, no. 10, pp. 2584-2596, 2013. 


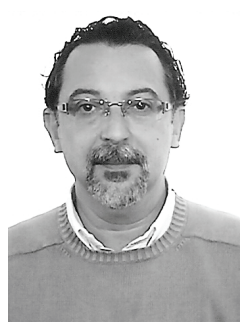

Manuel Delgado-Restituto (M'96, SM'12) received the M.S. degree in Physics and the Ph.D. degree (with honors) on Physics-Electronics from the University of Seville, Spain, in 1988 and 1996, respectively. Since the Doctorate, he has been working in the Institute of Microelectronics of Seville/CNMCSIC (IMSE-CNM/CSIC). In September 1999, he obtained a permanent position as CSIC Tenured Scientist (equivalent to Assistant Professor) and in June 2009, he upgraded to CSIC Research Scientist (equivalent to Associate Professor). Along this tenured professional period, he has directed 9 Ph.D. theses and 11 M.S. theses in the field of mixed-signal integrated circuits. Additionally, he has participated in some 20 National and European funded Research Projects, 7 of them with the role of main researcher. Besides, he has participated in 4 industrial contracts and realized several consultant services to industry. Currently, he heads a research group in IMSE-CNM/CSIC on low-power medical microelectronics and works in the design of silicon microsystems to understanding biological neural systems, the development of neural prostheses and brain-machine interfaces, the implementation of wireless Body Area Network transceivers and the realization of RFID transponders with biomedical sensing capabilities. Dr. Delgado-Restituto has coauthored the books "Device-Level Modeling and Synthesis of High-Performance Pipeline ADCs" (Springer, 2011) and "Ultra Low Power Transceiver for Wireless Body Area Networks" (Springer, 2013); more than 20 chapters in contributed books, including original tutorials on chaotic integrated circuits, design of data converters and chips for bioengineering and neuroscience; and some 150 articles in peerreview specialized publications. Dr. Delgado-Restituto served as an Associate Editor for the IEEE TRANSACTIONS ON CIRCUITS AND SYSTEMSII: EXPRESS BRIEFS (2006-07) and for the IEEE TRANSACTIONS ON CIRCUITS AND SYSTEMS-I: REGULAR PAPERS (2008-11). He served as Deputy Editor-in-Chief (2011-13) and as Editor-in-Chief (2014-15) for the IEEE JOURNAL ON EMERGING AND SELECTED TOPICS IN CIRCUITS AND SYSTEMS. He is currently Vice President for Publications of IEEE CAS (2016). He is in the committee of different international conferences and has served as technical program chair in different international IEEE conferences.

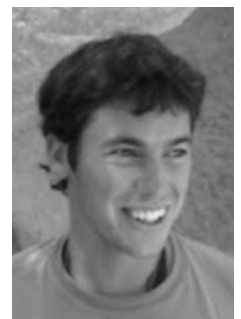

Alberto Rodríguez-Pérez (M'08) received the B.S degree in telecommunication engineering in and the $\mathrm{phD}$ degree from the University of Seville, Spain, in 2007 and 2013, respectively. From 2008 to 2012, he worked towards his $\mathrm{phD}$ in the Institute of Microelectronics of Seville, Spain. During that period, he has collaborated as a Visiting Scholar with the department of Electrical Engineering at the University of Montreal, Canada, in 2010 and with the Microelectronics Systems Laboratory at the EPFL, Switzerland, in 2011. He received a special recognition from the University of Sevilla at the end of his $\mathrm{phD}$ for the excellence of his research work. In 2013, he joined NXP Semiconductors in Eindhoven, The Netherlands, where he worked in the R+D department in the design of new and efficient topologies for high-speed serial interfaces transceivers for wireline communications. In 2014, he joined to KDPOF Ltd., a start-up company settled in Madrid, Spain, which develops transceivers for Gigabit Ethernet links over POF. There, he started and leads the Analog and Mixed Signal department, in charge of developing the optoelectronics and the analog front-end for the optical transceivers. He is also an Associate Professor in the University Carlos III of Madrid, Spain. His research interests cover from the design of low-power and low-voltage architectures for analog and mixedsignal circuits, power efficient solutions for biomedical sensor interfaces to the design of transceivers for high speed serial interfaces, high speed ADCs and DACs and optoelectronics for optical transceivers. He has authored and co-authored more than 30 papers in journals and peer-reviewed conferences, two book chapters and 7 patents. During his career, he has always tried to find the links between the academia, research and industry, providing innovative solutions for industrial products and developing research for new markets.

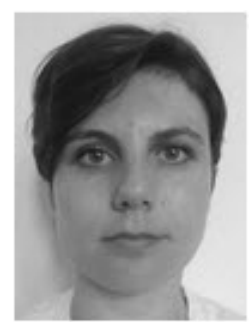

Angela Darie graduated in Electronics Engineering (specialization in Microtechnologies) in 2008 and got a M.Sc. degree in modern signal processing techniques in 2009 and Ph.D. degree in Electronics and telecommunications in 2011 from the Faculty of Electronics and Telecommunications, Gh. Asachi Technical University of Iasi, Romania. During the $\mathrm{Ph} . \mathrm{D}$. her main research focus has been on implementation of a wireless communication system based on FPGA and WSN motes to estimate a system's propagation characteristics through a propagation medium. Since 2012, she is an Associate Researcher at the Institute of Microelectronics of Seville, Spain. Her main research areas of interest are digital hardware design and interfaces for data acquisition and control.

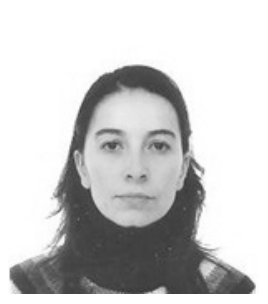

Cristina Soto-Sánchez received her academic Bachelor degree (University of Vigo, 1999) and her $\mathrm{PhD}$. degree in Biology (University of Santiago de Compostela, 2006). During her predoctoral education Dr. Soto-Sánchez got a broad background in electrophysiology and in the understanding of the somatosensory system processing. In September 2007, she got a postdoctoral position at the laboratory of Dr. Judith Hirsch, University of Southern California, USA, to study the early visual system. In 2011 , after her postdoctoral stay she moved back to Spain and joined the laboratory of Dr. Eduardo Fernández-Jover at the Bioengineering Institute of the University Miguel Hernández, as an experienced postdoctoral researcher belonging to the Network Research center in Bioengineering, Biomaterials and Nanomedicine, CIBER-BBN. Since then, she has been studying the properties of neural populations of visual cortex among other projects. She also maintains an active collaboration with the CIBER BBN-NanoBioCel Group to transfect brain neurons to treat genetic diseases. Besides, she actively collaborates with the Institute of Microelectronics of Seville/CNM-CSIC (IMSE-CNM/CSIC) in the biological aspects of the projects. As a result of all different projects and collaborations, Dr. Soto-Sánchez has published 16 peerreviewed scientific papers, 6 of them as first author, and several proceeding papers and communications to international and national conferences. Since 2012 she teaches as Associate Professor at the University of Alicante, Spain.

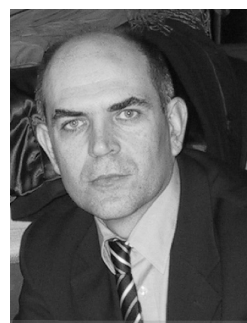

Eduardo Fernández-Jover received a M.D. degree from the University of Alicante (1986) and a Ph.D. in Neuroscience with honors in 1990. He is currently Full Professor and Chairman of the Department of Histology and Anatomy and Director of the Biomedical Neuroengineering Research Group (NBIO) of the University Miguel Hernández and CIBER BBN (Spain). He is a qualified MD who combines biomedicine (molecular and cellular biology, biochemistry, anatomy, physiology and regenerative medicine) with the physical sciences and engineering (mathematics, physics and applied chemistry) to better understand and safely interact with the nervous system. 


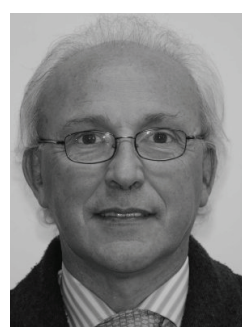

Ángel Rodríguez-Vázquez (F'96) received undergraduate and $\mathrm{PhD}$ degrees in Physics-Electronics with several national and international awards, including an IEEE award. After different research stays in University of California-Berkeley and Texas AM University he became a Full Professor of Electronics at the University of Sevilla in 1995 . He cofounded the Institute of Microelectronics of Sevilla, under the umbrella of the Spanish Council Research (CSIC) and the University of Sevilla and started a research group on Analog and Mixed-Signal Circuits for Sensors and Communications. In 2001 he was the main promotor and cofounder of the start-up company AnaFocus Ltd and served as CEO, on leave from the University, until June 2009, when the company reached maturity as a worldwide provider of smart CMOS imagers and vision systems-on-chip. His research is on the design of analog and mixed-signal front-ends for sensing and communication, including smart imagers, vision chips and low-power sensoryprocessing microsystems. He has authored 11 books, 36 additional book chapters, and some 150 journal articles in peer-review specialized publications. $\mathrm{He}$ has presented invited plenary lectures at different international conferences and has received a number of awards for his research (the IEEE GuilleminCauer best paper award, two Wiley's IJCTA best paper awards, two IEEE ECCTD best paper award, one SPIE-IST Electronic Imaging best paper award, the IEEE ISCAS best demo-paper award and the IEEE ICECS best demopaper award). He was elected Fellow of the IEEE for his contributions to the design of chaos-based communication chips and neuro-fuzzy chips. His research work got some 6,900 citations; he has an h-index of 42 and an i10index of 136 . He has always been looking for the balance between long-term research and innovative industrial developments. AnaFocus Ltd. was founded on the basis of his patents on vision chips and he participated in the foundation of the Hungarian start-up company AnaLogic Ltd. He has Eight Patents filed, three of which have been licensed to companies. He has served as Editor, Associate Editor and Guest Editor for different IEEE and non-IEEE journals, is in the committee of several international journals and conferences, and has chaired several international IEEE and SPIE conferences. He served as VP Region 8 of the IEEE Circuits and Systems Society (2009-2012) and as Chair of the IEEE CASS Fellow Evaluation Committee (2010, 2012, 2013, 2014 and 2015). 